\title{
Symmetry deduction from spectral fluctuations in complex quantum systems
}

\author{
S. Harshini Tekur* \\ Max-Planck-Institut für Physik komplexer Systeme, Nöthnitzer Straße 38, 01187 Dresden, Germany \\ M. S. Santhanam ${ }^{\dagger}$ \\ Indian Institute of Science Education and Research, Dr. Homi Bhabha Road, Pune 411 008, India
}

(Received 30 June 2020; accepted 17 August 2020; published 9 September 2020)

\begin{abstract}
The spectral fluctuations of complex quantum systems, in an appropriate limit, are known to be consistent with those obtained from random matrices. However, this relation between the spectral fluctuations of physical systems and random matrices is valid only if the spectra are desymmetrized. This implies that the fluctuation properties of the spectra are affected by the discrete symmetries of the system. In this Rapid Communication, it is shown that in the chaotic limit the fluctuation characteristics and symmetry structure for any arbitrary sequence of measured or computed levels can be inferred from its higher-order spectral statistics without desymmetrization. In particular, we consider a spectrum composed of $k>0$ independent level sequences with each sequence having the same level density. The $k$ th order spacing ratio distribution of such a composite spectrum is identical to its nearest-neighbor counterpart with a modified Dyson index $k$. This is demonstrated for the spectra obtained from random matrices, quantum billiards, spin chains, and experimentally measured nuclear resonances with disparate symmetry features.
\end{abstract}

DOI: 10.1103/PhysRevResearch.2.032063

Spectral fluctuations in complex quantum systems are analyzed using the theoretical framework of random matrix theory (RMT) in many areas of physics [1-5]. These include few-body systems studied in quantum chaos [6] to interacting many-body systems in condensed matter physics [7], nuclear physics [8], and atomic physics [9]. These fluctuations carry signatures of the distinct phases observed in the physical systems, viz., integrable or chaotic limit of the underlying classical system [10], metallic or insulating phase [11], localized or thermal phase of many-body systems [12], and low-lying shell model or mixing regime of nuclear spectra $[13,14]$. Indeed, the level spacing distribution of the desymmetrized eigenlevels is a popular diagnostic tool to discriminate between phases of physical systems in many areas of physics, and remarkably even outside of physics [4,15-18].

Beginning with the Wigner surmise [19] in the context of nuclear spectra, the present consensus is that the spectral fluctuations of complex quantum systems, in a suitable limit, display a level repulsion consistent with that of an appropriately chosen ensemble of random matrices. For the special case of quantum chaotic systems, the Bohigas-Giannoni-Schmidt (BGS) conjecture encapsulates this connection between the spectra from physical systems

\footnotetext{
*harshini.t@gmail.com

†santh@iiserpune.ac.in

Published by the American Physical Society under the terms of the Creative Commons Attribution 4.0 International license. Further distribution of this work must maintain attribution to the author(s) and the published article's title, journal citation, and DOI.
}

and random matrices [20]. This has been amply verified in experiments [21], simulations [22], and derives some theoretical support based on semiclassical techniques [23].

Discrete symmetries of the system, i.e., invariance of the potential under parity, reflection, and rotation, are crucial in realizing this connection between spectral fluctuations and dynamical phases. In the presence of symmetries, the Hilbert space of the system splits into invariant subspaces or the Hamiltonian matrix $\mathcal{H}$ becomes block diagonal, i.e., $\mathcal{H}=$ $\mathcal{H}_{1} \oplus \mathcal{H}_{2} \oplus \cdots \mathcal{H}_{m}$, with each block $\mathcal{H}_{i}, i=1,2, \ldots, m$ characterized by good quantum numbers corresponding to the respective symmetries [6]. This is schematically shown for chaotic billiards, with $m=4$ symmetry sectors, in Fig. 1. To compute any measure of spectral fluctuation, all the discrete levels must be drawn from the same subspace [shown as blocks in Fig. 1(a)]. If symmetries are ignored and levels from different blocks are superposed, as depicted in Fig. 1(c), the genuine correlation between levels (that should have produced level repulsion) is masked by near degeneracies resulting in level clustering. This effect becomes even more dominant as the number of superposed spectra $m$ increases. This is misleading since level clustering is also a spectral signature of integrable systems [24].

This implies that the level correlations are sensitive to the presence or absence of symmetries. It is then reasonable to expect that fluctuations of composite spectra, superposed from many independent blocks, contain information about the entire system's symmetry structure. However, any measure based on the nearest-neighbor (NN) fluctuations, such as the popular NN level spacing distribution, will always tend to the Poissonian limit (level clustering) due to the superposition of noninteracting blocks [25]. In this Rapid 


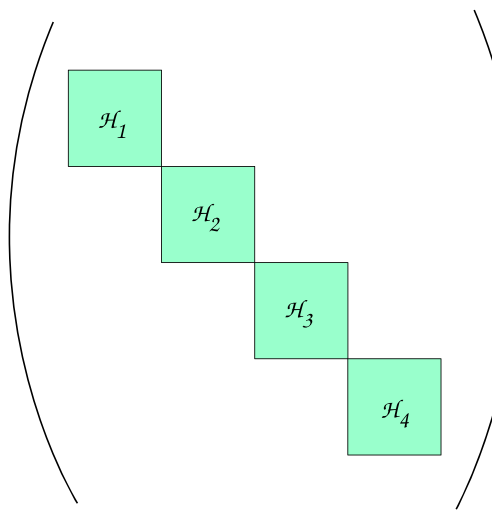

(a)

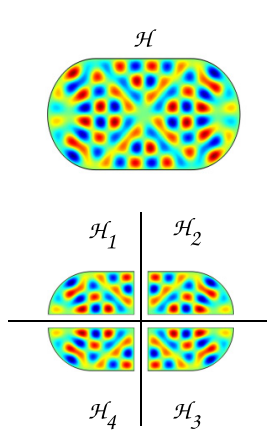

(b)

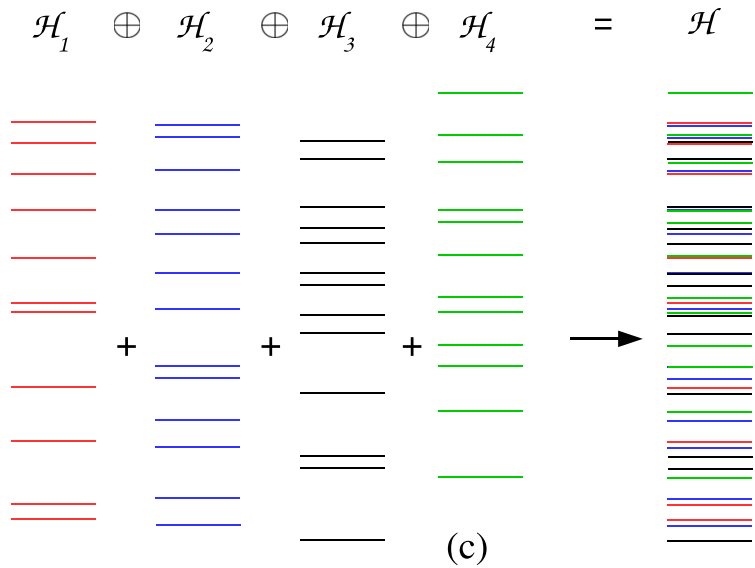

(c)

FIG. 1. (a) A schematic of the Hamiltonian matrix for stadium billiards with each block characterized by a good quantum number. (b) The potential for the stadium billiard with one of its eigenstates superposed on it. The bottom part shows a desymmetrized version of the same eigenstate indicating one possible relation to the blocks in the Hamiltonian in (a). (c) Schematic of the eigenlevels arising from each symmetry block, $\mathcal{H}_{1}$ (red colored levels) to $\mathcal{H}_{4}$ (green). The levels of $\mathcal{H}$ in the last column are a superposition of all these desymmetrized levels.

Communication, rigorous numerical evidence is presented to show that the higher-order level spacing ratio not only identifies the true fluctuation character, viz., level clustering or repulsion, but also allows us to deduce quantitative information about the symmetry structure of the composite Hamiltonian matrix $\mathcal{H}$.

This result obviates the need for symmetry decomposition of quantum systems, allows for the analysis of any arbitrary sequence of experimentally observed levels with an unknown symmetry structure, and is also of considerable interest in RMT [26]. Let $G$ be a random matrix such that $G=G_{1} \oplus$ $G_{2} \oplus \cdots G_{m}$, a superposition of $m$ blocks, each of which is a Gaussian random matrix with an identical level density. Given an arbitrary sequence of eigenvalues of $G$, the fluctuation properties and the block structure of $G$ can be inferred from its higher-order fluctuation statistics. The proposed method is straightforward, involving only the calculation of spacing ratios. This is in contrast to the cumbersome methods proposed earlier based on the two-level cluster function and requiring regression to deduce $m$ from any composite spectrum $[1,2,19,27]$, all of which require unfolding as the first step.

Consider a sequence of eigenvalues $E_{i}, i=1,2, \ldots, N$ of a quantum operator or a random matrix. Spectral fluctuations are relatively easier to analyze using spacing ratios defined as $r_{i}=\frac{E_{i+2}-E_{i+1}}{E_{i+1}-E_{i}}, i=1,2, \ldots, N-2$ [28], as spacing ratios are independent of the local density of states and hence do not require spectral unfolding. For random matrix ensembles with a Dyson index $\beta=1,2$, and 4 , corresponding respectively to the Gaussian orthogonal, unitary, and symplectic ensembles, the distribution of spacing ratios is given by [29]

$$
P(r, \beta)=C_{\beta} \frac{\left(r+r^{2}\right)^{\beta}}{\left(1+r+r^{2}\right)^{1+3 \beta / 2}},
$$

where $C_{\beta}$ is a constant, as listed in Ref. [29]. These RMT models are applicable to Hamiltonians with time-reversal invariance (TRI) $(\beta=1)$, without TRI $(\beta=2)$, and TRI with spin- $1 / 2$ interactions $(\beta=4)$. The main focus of this Rapid
Communication is on RMT models applicable to Hamiltonians with TRI $(\beta=1)$. For integrable systems, the ratio distribution becomes $P_{P}(r)=1 /(1+r)^{2}$. Recently, an expression for the nearest-neighbor spacing ratio distribution has been obtained taking into account the spectral transition from integrable to chaotic limits, and also crossovers from one symmetry class to another [30].

As motivation, in Fig. 2, the numerically computed distribution of $\mathrm{NN}$ spacing ratios $P(r)$ is shown for circular (integrable) [31] and stadium (chaotic) [32] billiards. The integrable billiards [Fig. 2(a)] expectedly agree with $P_{P}(r)$. Note that the stadium billiard has $C_{2 v}$ point group symmetry with four irreducible representations (irreps). If the spectra from each irrep are analyzed separately, by BGS conjecture, an agreement with $P(r, 1)$ of GOE is observed [Fig. 2(c)]. However, in Fig. 2(b), the spectra from all the irreps are superposed, and hence the ratio distribution is closer to $P_{P}(r)$ with a pronounced deviation from $P(r, 1)$. In quantum systems with a chaotic limit, as demonstrated below, the true character of their spectral fluctuations and the number $m$ of independent spectra superposed can all be inferred using only

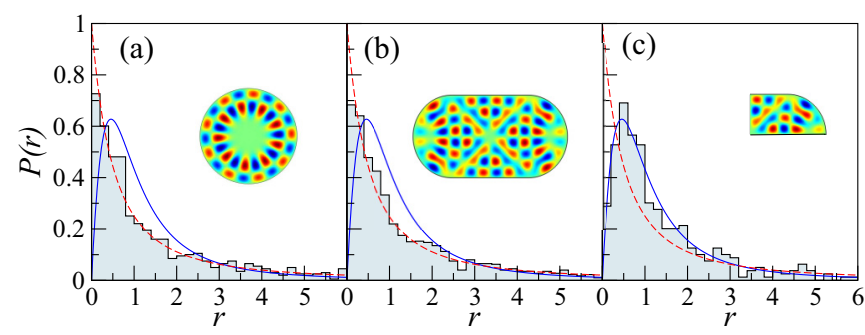

FIG. 2. Distribution of the NN spacing ratios (histograms) $P(r)$ for the (a) circular, (b) stadium, and (c) desymmetrized stadium billiards. The dashed (red) line is $P_{P}(r)$ and the solid (blue) curve is the Wigner surmise for ratios. The inset shows the shape of the billiards and a typical eigenfunction superposed on it to emphasize its symmetry structure. 
the higher-order spacing ratio (HOSR) distributions without $a$ priori knowledge of its symmetry structure.

To this end, we consider a nonoverlapping $k$ th order spacing ratio, defined as

$$
r_{i}^{(k)}=\frac{s_{i+k}^{(k)}}{s_{i}^{(k)}}=\frac{E_{i+2 k}-E_{i+k}}{E_{i+k}-E_{i}}, \quad i, k=1,2,3, \ldots
$$

In what follows, spectra from $m$ independent blocks are superposed, and their distribution of $k$ th order spacing ratios are denoted by $P^{k}(r, \beta, m)$. We consider only $\beta=1$. For the special case involving NN ratios, we denote $P^{1}(r, \beta, 1)=$ $P(r, \beta)$. The motivation for considering higher-order fluctuation statistics arises from a seminal result conjectured in Ref. [33] and proved by Gunson [34] for the case of circular ensembles of RMT. If two independent spectra from the circular orthogonal ensemble (COE) are superposed, upon integrating out every alternate eigenvalue, the joint probability distribution of the remaining eigenvalues follows circular unitary ensemble (CUE) statistics. In terms of higher-order measures, this result states that the second-order statistics of two superposed COE spectra converges to $\mathrm{NN}$ statistics of CUE. This is reflected in the distribution of spacings and spacing ratios as well. In the limit of large matrix dimensions, this result holds for Gaussian ensembles, too, yielding $P^{2}(r, 1,2)=P(r, 2)$ for two superposed spectra. If the order of each of the $m$ GOE matrices is the same, then this may be generalized for the superposition of $m$ GOE spectra as

$$
P^{k}(r, 1, m)=P\left(r, \beta^{\prime}\right),
$$

where

$$
\beta^{\prime}=m=k,
$$

implying that its $k$ th order spacing ratio distribution converges to NN statistics $P\left(r, \beta^{\prime}\right)$ with $\beta^{\prime}=k$. Equation (3) is the main result of this Rapid Communication, and is well supported by numerical experiments involving a small number of mixed symmetries (up to $m=5$ ). In contrast, irrespective of how many uncorrelated spectra are superposed corresponding to integrable systems, the $k$ th order spacing ratio distribution can be obtained (details in the Supplemental Material [35]) as

$$
P_{P}^{k}(r)=\frac{(2 k-1) !}{[(k-1) !]^{2}} \frac{r^{k-1}}{(1+r)^{2 k}} .
$$

For $k=1$, this reduces to $\frac{1}{(1+r)^{2}}$, the correct limit for the NN spacing ratio for uncorrelated spectra. We note that Eq. (3) is reminiscent of a scaling relation reported recently in Ref. [36].

For the superposition of $m=2-5$ independent GOE spectra, the validity of Eq. (3) is verified in Fig. 3. In this figure, an excellent agreement is observed between histograms obtained from the computed eigenvalues of GOE matrices and the solid line representing $P\left(r, \beta^{\prime}=k\right)$. For uncorrelated eigenvalues, a similar agreement with Eq. (4) is observed. In order to independently obtain a best quantitative estimate for $\beta^{\prime}$ in Eq. (3) for a given superposition of $m$ spectra, we compute

$$
D\left(\beta^{\prime}\right)=\sum_{i}\left|I_{\mathrm{obs}}^{m}\left(r_{i}, 1, m\right)-I\left(r_{i}, \beta^{\prime}\right)\right| .
$$

In this, $I_{\mathrm{obs}}^{m}(r, 1, m)$ and $I\left(r, \beta^{\prime}\right)$ represent the cumulative distribution functions corresponding respectively to the observed

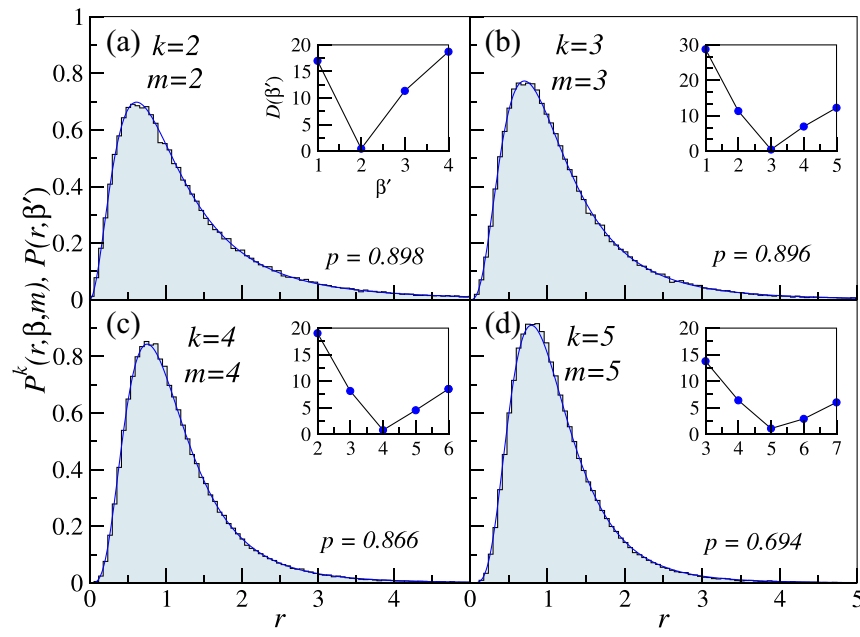

FIG. 3. Distribution of $k$ th order spacing ratios (histograms) for a superposition of $m$ GOE spectra, each obtained from a matrix of order $N=40000$, shown for $m=2-5$. The solid curve is $P\left(r, \beta^{\prime}\right)$, with $\beta^{\prime}=k$. The insets show $D(\beta)$ whose minimum correctly coincides with the expected value of $m$. The $p$ values from the KS test are also given.

histogram $P^{k}(r, 1, m)$ and the postulated function $P\left(r, \beta^{\prime}\right)$. If the minima of $D\left(\beta^{\prime}\right)$ occur at, say, $\beta^{\prime}=\beta_{0}$, then $\beta_{0}$ is the best estimate consistent with the observed data. As seen in the insets of Fig. 3, the minima in $D\left(\beta^{\prime}\right)$ coincide with the value of $m$, the number of superposed spectra. This is further corroborated by the Kolmogorov-Smirnov (KS) test [37] at a significance level of 0.05 for each case. The $p$ value, as anticipated, is maximum at the same value of $m$.

A complete picture is revealed in Fig. 4 for a superposition of $m=4$ independent GOE spectra, where the computed

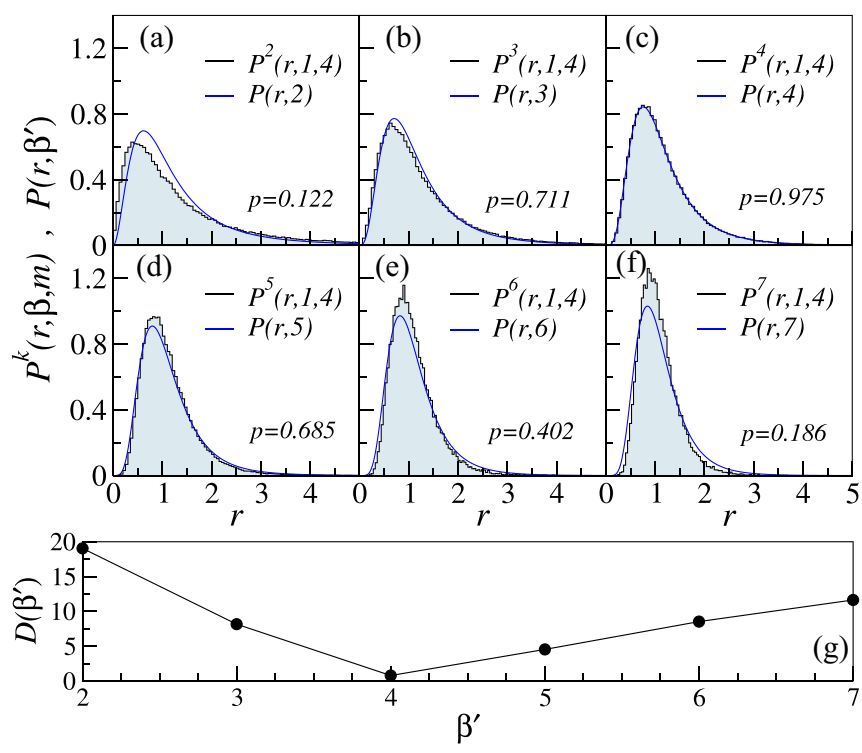

FIG. 4. (a)-(f) Computed $k$ th order spacing ratio distribution (histogram) for superposed spectra from four GOE matrices of order $N=40000$. Note that the best agreement with $P\left(r, \beta^{\prime}=k\right)$ (blue line) obtained only for $\beta^{\prime}=k=4$, and is confirmed by the $p$ value from the KS test, displaying maxima at $\beta^{\prime}=4$. (g) $D\left(\beta^{\prime}\right)$ vs $\beta^{\prime}$ has minima at $\beta^{\prime}=4$. Both $D\left(\beta^{\prime}\right)$ and $p$ indicate the validity of Eq. (3). 


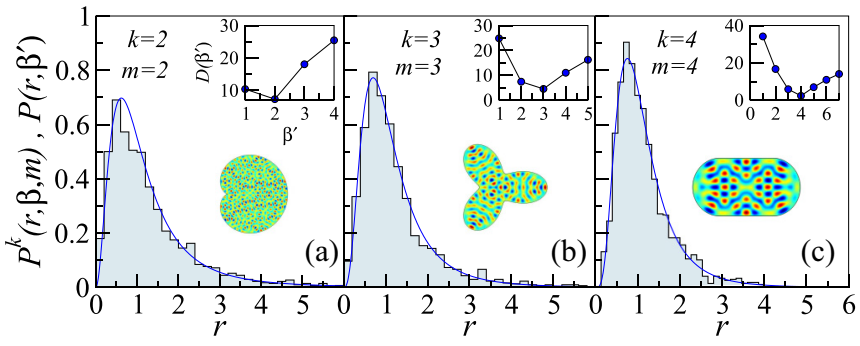

FIG. 5. HOSR distribution (histogram) for the billiards family in which spectra from (a) $k=2$, (b) $k=3$, and (c) $k=4$ irreps are superposed. The higher-order distributions are consistent with $P\left(r, \beta^{\prime}\right)$, and $\beta^{\prime}=k$ as dictated by Eq. (3). The insets display $D\left(\beta^{\prime}\right)$ and its minimum indicates the correct number of irreps in the system. Also shown is the shape of the billiards with an arbitrarily chosen chaotic eigenstate to highlight its symmetry.

histogram for the $k$ th order ratio is shown for $k=2-7$. Based on Eq. (3), we expect it to be consistent with $P\left(r, \beta^{\prime}=4\right)$. For each $k, P^{k}(r, 1,4)$ is matched against the corresponding $P\left(r, \beta^{\prime}\right)$, and $D\left(\beta^{\prime}\right)$ is calculated. Both visually and quantitatively [the minima of $D\left(\beta^{\prime}\right)$ in Fig. 4(e)], the best agreement is observed for $k=4$, verifying the main result in Eq. (3). Significantly, for the superposed spectra, Eqs. (3) and (4) can be used to infer the correct nature of the spectral fluctuations (level repulsion or clustering) and also to determine the number of superposed independent blocks for a random matrix or the number of diagonal blocks in the Hamiltonian matrix of a complex quantum system, if the system is chaotic. In what follows, the result in Eqs. (3) and (4) will be applied to chaotic systems possessing different symmetries, notably billiards and spin chains, and most importantly to the experimentally measured data of nuclear resonances.

First, we consider quantum billiards, in which a free particle is confined in a cavity defined by a variety of boundaries [38], whose eigenspectrum is obtained by solving the Helmholtz equation with Dirichlet boundary conditions. They are popular models in Hamiltonian chaos and mesoscopic physics [39] and have experimentally realized variants [40]. Modifying the boundary or shape of the billiard changes its symmetry and also drives it from integrability to chaos. For a billiard whose boundary is parametrized by $r(\phi)=r_{0}(1+$ $\epsilon \cos \phi)$, as $\epsilon$ varies from 0 to 1 , the system transitions from integrable to chaotic dynamics. For $\epsilon=0$, a circular billiard shown in Fig. 2(a) is obtained. This is an integrable system and its higher-order spacings are in agreement with Eq. (4) (see Supplemental Material [35]). For $\epsilon=1$, the cardioid billiard is obtained [41], possessing two irreps due to reflection symmetry about the horizontal axis. Thus, eigenlevels obtained disregarding symmetry would correspond to a superposition of two GOE spectra. As anticipated by Eq. (3), its second-order distribution $P^{2}(r, 1,2)$ is consistent with $P(r, 2)$ [Fig. 5(a)]. A billiard with three irreps, similar in shape to the one that has been experimentally realized [42], is obtained by parametrizing its boundary as $r(\phi)=r_{0}[1+0.3 \cos (3 \phi)]$. This model, with symmetries ignored and after removing degeneracies arising from the two-dimensional irreps, corresponds to a superposition of three chaotic spectra and the best match for $P^{3}(r, 1,3)$ is provided by $P(r, 3)$ [Fig. 5(b)].

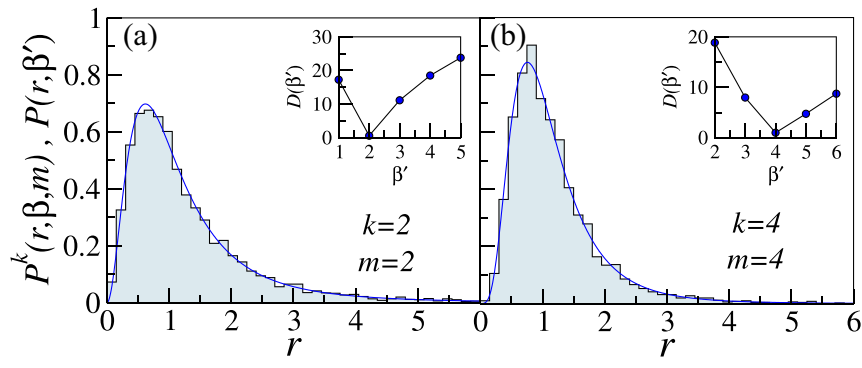

FIG. 6. HOSR distribution computed for the spin-1/2 chain Hamiltonian in Eq. (6), with (a) an odd number of sites with two irreps and (b) an even number of sites with four irreps. The insets show $D\left(r, \beta^{\prime}\right)$ and its minima identify the number of irreps.

A chaotic billiard with four irreps is the well-studied Bunimovich stadium billiard [43] shown in Fig. 5(c). This has reflection symmetry about the $x$ and $y$ axes and, in accordance with Eq. (3), $P^{k}(r, 1,4)$ displays the best correspondence with $P\left(r, \beta^{\prime}\right)$ for $k=\beta^{\prime}=4$ [Fig. 5(c)]. For all of these cases, insets in Fig. 5 show that the minima of $D\left(\beta^{\prime}\right)$ coincide with $\beta^{\prime}=k$, the number of irreps.

Next, a spin-1/2 chain with the Hamiltonian [44]

$$
\begin{aligned}
H= & \sum_{i=1}^{L-1}\left[J_{x y}\left(S_{i}^{x} S_{i+1}^{x}+S_{i}^{y} S_{i+1}^{y}\right)+J_{z} S_{i}^{z} S_{i+1}^{z}\right] \\
& +\eta \sum_{i=1}^{L-2}\left[J_{x y}^{\prime}\left(S_{i}^{x} S_{i+2}^{x}+S_{i}^{y} S_{i+2}^{y}\right)+J_{z}^{\prime} S_{i}^{z} S_{i+2}^{z}\right]
\end{aligned}
$$

is considered, where $L$ is the number of sites, $J_{x y}$ and $J_{z}$ are the NN coupling strengths in three directions (coupling along $x$ and $y$ being the same), and $J_{x y}^{\prime}$ and $J_{z}^{\prime}$ are the next-NN coupling strengths. This system is integrable for $\eta=0$ (as shown in Fig. 1 in the Supplemental Material [35]), and chaotic for $\eta \gtrsim 0.2$. The total spin in the $z$ direction, $S_{z}$, is conserved and the Hamiltonian is block diagonal in the $S_{z}$ basis, each block corresponding to a given value of $S_{z}$. However, other symmetries that exist in this system would erroneously lead to fluctuation statistics appearing to be integrable in this subspace (not shown here). For an odd number of sites $\left(L_{\text {odd }}\right)$, on computing the HOSRs and comparing with corresponding $P\left(r, \beta^{\prime}\right), k=\beta^{\prime}=2$ has the best match [Fig. 6(a)]. But for an even number of sites $\left(L_{\text {even }}\right)$, HOSRs correspond to $k=$ $\beta^{\prime}=4$ [Fig. 6(b)]. This is because for $L_{\text {odd }}$ or $L_{\text {even }}$, the parity operator (with eigenvalues \pm 1 ) commutes with $H$, leading to two invariant subspaces in a given $S_{z}$ block. For $L_{\text {even }}$, an additional rotational symmetry exists (with eigenvalues \pm 1 ) for the corresponding operator, giving rise to four irreps. The other parameters used in Figs. 6(a) and 6(b) are $J_{x y}=$ $J_{x y}^{\prime}=1.0, J_{z}=J_{z}^{\prime}=0.5$, with $L_{\text {even }}=14$ and $L_{\text {odd }}=15$. For these systems, the KS test results given in the Supplemental Material [35] provide yet another verification of the scaling relation.

Even for systems whose Hamiltonian is not well defined or unknown as in the case of complex nuclei, experimentally observed nuclear resonance data can be analyzed to characterize their fluctuation statistics and find their number of irreps. It is assumed that the system being observed is in the putative regime in which RMT results can be applied. We consider a 

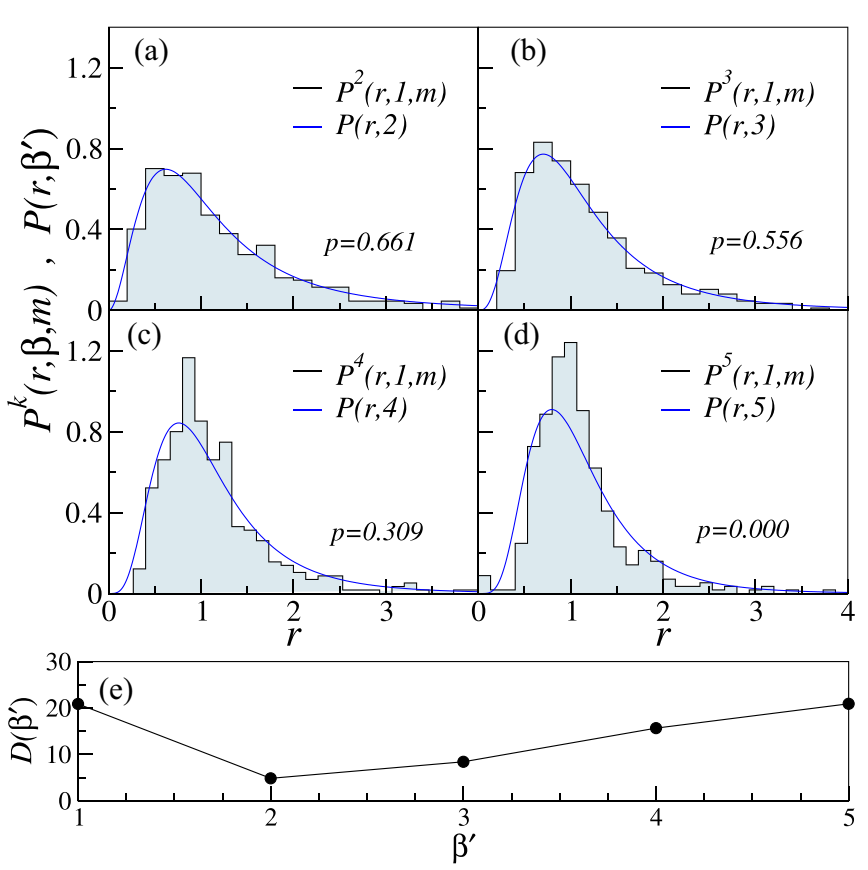

FIG. 7. (a)-(d) The $k$ th order spacing ratio distribution (histogram) for experimentally observed nuclear resonances for tantalum $\left(\mathrm{Ta}^{181}\right)$, showing the best correspondence for $k=2$. The dashed line is $P\left(r, \beta^{\prime}=k\right)$. The $p$ value from the $\mathrm{KS}$ test shows a maximum at $\beta^{\prime}=2$. (e) $D\left(\beta^{\prime}\right)$ shows minima at $\beta^{\prime}=2$, reinforcing the validity of Eq. (3).

sequence of experimentally observed neutron resonances for a $\mathrm{Ta}^{181}$ nucleus [45] whose NN spacing distribution, discussed in Ref. [14], does not match the Wigner surmise. On calculating HOSR distributions, remarkably, Eq. (3) holds well for $k=2$, further confirmed by the minima of $D\left(\beta^{\prime}\right)$ for $\beta^{\prime}=2$ in Fig. 7, and the corresponding KS test results. This indicates the presence of two independent symmetry sectors, and is indeed the case, as confirmed in Refs. [14,45]. This measured sequence consists of a superposition of levels having angular momenta $J=3$ and 4 , and when symmetry decomposed, are in broad agreement with the Wigner surmise. Clearly, for an arbitrary sequence of measured levels, if the random matrix description is valid, HOSRs based on Eq. (3) can unambiguously identify the true fluctuation character and the number of symmetry sectors.

In experiments, often measurement errors lead to missing levels [46] and hence incorrect identification of the fluctuation character and number of irreps. The robustness of Eq. (3) to missing levels in a superposition of GOE spectra was tested in two ways: (a) by randomly deleting levels, and (b) by preferentially deleting one of a pair that is nearly degenerate. Upon computing $D\left(\beta^{\prime}\right)$ in each case (details in the Supplemental Material [35]), the scaling in Eq. (3) holds well even if $20-30 \%(40 \%)$ of the levels are removed through random deletions (deletion of near-degenerate levels). This is because higher-order fluctuations are unaffected by, and hence largely insensitive to, randomly missing levels. This virtue is inherent to this method and has practical significance for analyzing experimental data.

To summarize, quantum systems must be symmetry decomposed to reveal their true spectral fluctuation characteristics. This also implies that the fluctuations carry symmetry information, though extracting it unambiguously from NN fluctuation statistics is nontrivial. As demonstrated in this Rapid Communication, the higher-order spacing ratio distributions can reveal, apart from the fluctuation characteristics, quantitative information about the symmetry structure. For a superposition of $k$ independent spectra (with identical level densities) drawn from an ensemble of RMT, the central result [Eq. (3)] relates the $k$ th order spacing ratio distribution for random matrices with a Dyson index $\beta=1$ to the corresponding nearest-neighbor statistics with $\beta^{\prime}=k$. For quantum systems in the classically chaotic limit and in the regime of applicability of Wigner-Dyson ensembles of RMT, this elegant relation determines the number of irreps (or diagonal blocks) present in a Hamiltonian matrix. This is exploited to analyze any arbitrary sequence of experimentally measured or computed levels, even if the system's Hamiltonian and symmetry structure are unknown. This technique requires neither unfolding nor a free-parameter estimation, nor the computation of cumbersome correlation or power spectral functions and hence is straightforward to implement. Further, for uncorrelated eigenvalues, the HOSR distribution has been derived and can be used as a test of integrability. These results are demonstrated using disparate physical systems such as quantum billiards, spin chains, and experimentally measured nuclear resonances. It must be remarked that just as the Bohigas-Giannoni-Schmidt conjecture [20] is valid for quantum systems in their chaotic limit, the results presented here too are strictly valid in the same regime in which RMT is applicable. In principle, this approach may be extended to weakly chaotic or mixed systems (outside of the RMT regime) by considering a broader class of higher-order spacing ratios, and these results will be reported elsewhere.

M.S.S. would like to acknowledge the financial support from SERB through MATRICS grant MTR/2019/001111.
[1] T. Guhr, A. Muller-Groeling, and H. A. Weidenmuller, Phys. Rep. 299, 189 (1998).

[2] C. E. Porter, Statistical Theories of Spectra: Fluctuations (Academic, New York. 1965).

[3] F. Haake, Quantum Signatures of Chaos (Springer, Berlin, 2013).

[4] The Oxford Handbook of Random Matrix Theory, edited by G. Akemann, J. Baik, and P. Di Francesco (Oxford University Press, New York, 2011).
[5] P. J. Forrester, Log-Gases and Random Matrices (LMS-34) (Princeton University Press, Princeton, NJ, 2010).

[6] H. J. Stöckmann, Quantum Chaos: An Introduction (Cambridge University Press, Cambridge, UK, 2000).

[7] C. W. Beenakker, Rev. Mod. Phys. 69, 731 (1997); Y. Alhassid, ibid. 72, 895 (2000).

[8] J. M. G. Gómez, K. Kar, V. K. B. Kota, R. A. Molina, A. Relaño, and J. Retamosa, Phys. Rep. 499, 103 (2011). 
[9] N. Rosenzweig and C. E. Porter, Phys. Rev. 120, 1698 (1960); H. S. Camarda and P. D. Georgopulos, Phys. Rev. Lett. 50, 492 (1983).

[10] L. Reichl, The Transition to Chaos: Conservative Classical Systems and Quantum Manifestations (Springer, Berlin, 2013).

[11] H. Hasegawa and Y. Sakamoto, Prog. Theor. Phys. Suppl. 139, 112 (2000); S. M. Nishigaki, Phys. Rev. E 59, 2853 (1999).

[12] S. D. Geraedts, R. Nandkishore, and N. Regnault, Phys. Rev. B 93, 174202 (2016).

[13] H. A. Weidenmüller and G. E. Mitchell, Rev. Mod. Phys. 81, 539 (2009).

[14] T. A. Brody, J. Flores, J. B. French, P. A. Mello, A. Pandey, and S. S. Wong, Rev. Mod. Phys. 53, 385 (1981).

[15] J. Kwapien and S. Drozdz, Phys. Rep. 515, 115 (2012).

[16] M. S. Santhanam and P. K. Patra, Phys. Rev. E 64, 016102 (2001).

[17] L. Laloux, P. Cizeau, J.-P. Bouchaud, and M. Potters, Phys. Rev. Lett. 83, 1467 (1999); V. Plerou, P. Gopikrishnan, B. Rosenow, L. A. Nunes Amaral, and H. E. Stanley, ibid. 83, 1471 (1999).

[18] R. Couillet and M. Debbah, Random Matrix Methods for Wireless Communications (Cambridge University Press, Cambridge, UK, 2011).

[19] M. L. Mehta, Random Matrices (Academic, New York, 2004).

[20] O. Bohigas, M. J. Giannoni, and C. Schmit, Phys. Rev. Lett. 52, 1 (1984).

[21] D. Delande and J. C. Gay, Phys. Rev. Lett. 57, 2006 (1986); H. Friedrich and H. Wintgen, Phys. Rep. 183, 37 (1989); C. Ellegaard, T. Guhr, K. Lindemann, J. Nygard, and M. Oxborrow, Phys. Rev. Lett. 77, 4918 (1996).

[22] H. U. Baranger and P. A. Mello, Phys. Rev. Lett. 73, 142 (1994); J. N. Bandyopadhyay and A. Lakshminarayan, ibid. 89, 060402 (2002); Ph. Jacquod, H. Schomerus, and C. W. J. Beenakker, ibid. 90, 207004 (2003).

[23] S. Muller, S. Heusler, P. Braun, F. Haake, and A. Altland, Phys. Rev. Lett. 93, 014103 (2004); Phys. Rev. E 72, 046207 (2005).

[24] M. V. Berry and M. Tabor, Proc. R. Soc. London, Ser. A 356, 375 (1977).

[25] M. V. Berry and M. Robnik, J. Phys. A 17, 2413 (1984).

[26] P. J. Forrester and E. M. Rains, Probab. Theory Relat. Fields 130, 518 (2004).

[27] T. Guhr and H. A. Weidenmüller, Chem. Phys. 146, 21 (1990); L. Leviandier, M. Lombardi, R. Jost, and J. P. Pique, Phys. Rev. Lett. 56, 2449 (1986); J. B. French, V. K. B. Kota, A. Pandey, and S. Tomsovic, Ann. Phys. (N.Y.) 181, 198 (1988); R. A. Molina, J. Retamosa, L. Muñoz, A. Relaño, and E. Faleiro, Phys. Lett. B 644, 25 (2007).
[28] V. Oganesyan and D. A. Huse, Phys. Rev. B 75, 155111 (2007).

[29] Y. Y. Atas, E. Bogomolny, O. Giraud, and G. Roux, Phys. Rev. Lett. 110, 084101 (2013).

[30] A. L. Corps and A. Relaño, Phys. Rev. E 101, 022222 (2020).

[31] R. W. Robinet, Am. J. Phys. 64, 440 (1996).

[32] S. W. McDonald and A. N. Kaufman, Phys. Rev. Lett. 42, 1189 (1979); G. Casati, F. Valz-Gris, and I. Guarnieri, Lett. Nuovo Cimento 28, 279 (1980).

[33] F. J. Dyson, J. Math. Phys. 3, 1 (1962).

[34] J. Gunson, J. Math. Phys. 3, 752 (1962).

[35] See Supplemental Material at http://link.aps.org/supplemental/ 10.1103/PhysRevResearch.2.032063 for a detailed derivation of HOSR for integrable systems and further information on superposed spectra having missing levels.

[36] S. H. Tekur, U. T. Bhosale, and M. S. Santhanam, Phys. Rev. B 98, 104305 (2018).

[37] S. Teukolsky, W. H. Press, W. T. Vetterling, and B. P. Flannery, Numerical Recipes (Cambridge University Press, Cambridge, UK, 2007).

[38] M. Robnik, J. Phys. A: Math. Gen. 17, 1049 (1984).

[39] M. V. Berry, Eur. J. Phys. 2, 91 (1981); Ann. Phys. 131, 163 (1981).

[40] J. Stein and H.-J. Stöckmann, Phys. Rev. Lett. 68, 2867 (1992); A. Kudrolli, V. Kidambi, and S. Sridhar, ibid. 75, 822 (1995); P. So, S. M. Anlage, E. Ott, and R. N. Oerter, ibid. 74, 2662 (1995); H. Schanze, H.-J. Stöckmann, M. Martínez-Mares, and C. H. Lewenkopf, Phys. Rev. E 71, 016223 (2005); B. Dietz, T. Friedrich, H. L. Harney, M. Miski-Oglu, A. Richter, F. Schäfer, and H. A. Weidenmüller, Phys. Rev. Lett. 98, 074103 (2007).

[41] A. Bäcker, F. Steiner, and P. Stifter, Phys. Rev. E 52, 2463 (1995).

[42] B. Dietz, A. Heine, V. Heuveline, and A. Richter, Phys. Rev. E 71, 026703 (2005).

[43] H. Alt, C. Dembowski, H.-D. Gräf, R. Hofferbert, H. Rehfeld, A. Richter, and C. Schmit, Phys. Rev. E 60, 2851 (1999).

[44] A. Gubin and L. F. Santos, Am. J. Phys. 80, 246 (2012).

[45] G. Hacken, R. Werbin, and J. Rainwater, Phys. Rev. C 17, 43 (1978).

[46] M. Ławniczak, M. Białous, V. Yunko, S. Bauch, and L. Sirko, Phys. Rev. E 98, 012206 (2018); M. Białous, V. Yunko, S. Bauch, M. Ławniczak, B. Dietz, and L. Sirko, Phys. Rev. Lett. 117, 144101 (2016); O. Bohigas and M. P. Pato, Phys. Lett. B 595, 171 (2004); F. J. Dyson and M. L. Mehta, J. Math. Phys. 4, 701 (1963). 